\title{
RACISMO ESTRUTURAL: IMPLICAÇÕES NO PROCESSO DE TRABALHO DO ENFERMEIRO NA ATENÇÃO PRIMÁRIA À SAÚDE
}

STRUCTURAL RACISM: IMPLICATIONS ON THE WORK PROCESS OF NURSES IN PRIMARY HEALTH CARE

RACISMO ESTRUCTURAL: IMPLICACIONES EN EL PROCESO LABORAL DEL ENFERMERO EN LA ATENCIÓN PRIMARIA A LA SALUD

Maísa Galdino Pereira ${ }^{1}$

Daniele Pereira Soares 2

Cícera Renata Diniz Vieira Silva ${ }^{3}$

Dayze Djanira Furtado de Galiza 4

Mayara Evangelista de Andrade ${ }^{5}$

Marcelo Costa Fernandes 6

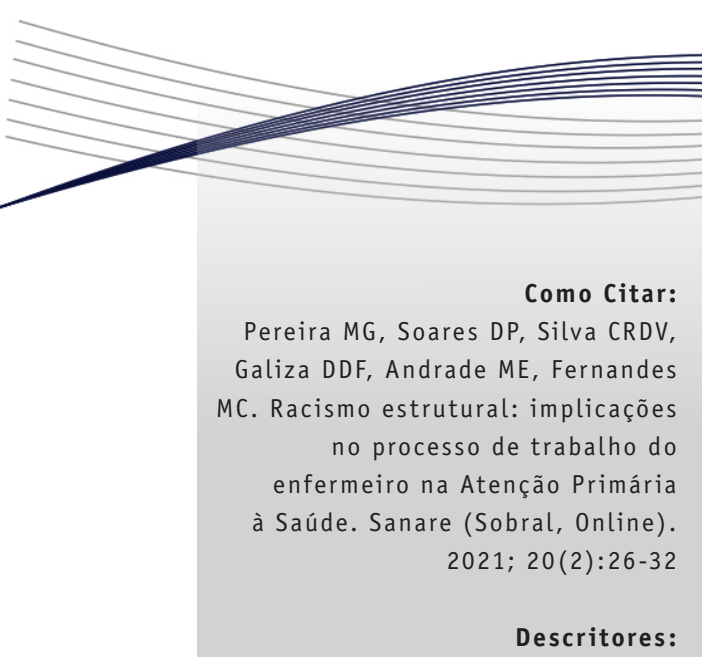

Saúde das Minorias Étnicas; Cuidados

de enfermagem; Atenção Primária à Saúde.

Descriptors:

Health of Ethnic Minorities; Nursing Care; Primary Health Care.

Descriptores:

Salud de Las Minorías Étnicas; Cuidados de enfermería; Atención Primaria a la salud.

\section{RESUMO}

Este estudo objetivou analisar o discurso do profissional enfermeiro acerca das implicações no processo de trabalho ofertado à população negra. Tratase de um estudo descritivo, com abordagem qualitativa, realizado entre os meses de março e maio de 2017, sustentado nos marcos conceituais da análise do discurso de Michel Pêcheux, realizado com oito enfermeiros da Atenção Primária à Saúde do município de Cajazeiras, Paraíba, Brasil. Para a coleta de dados, utilizou-se uma entrevista semiestruturada de maneira individual. A coleta ocorreu somente após a aprovação do Comitê de Ética em Pesquisa. Os enfermeiros apresentaram em seu corpus discursivo que os processos discriminatórios se destacam a partir de formas diversas, em especial por meio do preconceito e racismo e, ainda, pela associação das vulnerabilidades com a população negra. Dessa maneira, é fundamental a implementação de intervenções educativas sobre a saúde da população negra para os profissionais, rompendo barreiras histórico-sociais e fortalecendo a promoção da saúde.

Submetido: 04/03/2021

Aprovado: $17 / 11 / 2021$

Autor(a) para Correspondência: Daniele Pereira Soares Faculdade de Ciências Médicas da Paraíba

Endereço: Rua Francisco Beltrão, 90. Jardim Oceania. João Pessoa, Paraíba.

CEP: 58.037-605 E-mail: dani.b.d.d@hotmail.com
1. Enfermeira. Especialista em Enfermagem Clínica e em Saúde da Família. Universidade Federal de Campina Grande. E-mail: maisaenf.art@gmail.com ORCID: https://orcid.org/0000-0003-4692-5626

2. Enfermeira. Residente pelo Programa de Residência Multiprofissional em Saúde da Família e Comunidade. Faculdade de Ciências Médicas da Paraíba. E-mail: dani.b.d.d@hotmail.com ORCID: https://orcid.org/0000-0001$\underline{8575-5880}$

3. Enfermeira. Doutoranda em Ciências da Saúde pela Universidade Federal do Rio Grande do Norte. Universidade Federal de Campina Grande. E-mail: renatadiniz_enf@yahoo.com.br ORCID: https://orcid.org/0000-0002-0928$\underline{8368}$

4. Enfermeira. Doutoranda em Saúde Coletiva pela Universidade Estadual do Ceará. Universidade Federal de Campina Grande. E-mail: dayze_galiza@hotmail.com ORCID: https://orcid.org/0000-0001-9237-0372

5. Enfermeira. Mestra em Cuidado em Enfermagem e Saúde da Universidade Federal da Paraíba. Universidade Federal da Paraíba. E-mail: mayaraeandrade@hotmail.com 0RCID: https://orcid.org/0000-0001-5256-2169

6. Enfermeiro. Doutor em Cuidados Clínicos em Enfermagem e Saúde pela Universidade Estadual do Ceará. Universidade Federal de Campina Grande.E-mail: celo_cf@hotmail.com ORCID: https://orcid.org/0000-0003-1626$\underline{3043}$

Cert. de Redação Científica: Central das Revisões. Edição de texto: Karina Maria M. Machado. Revisão de provas: Texto definitivo validado pelos (as) autores(as). 


\section{ABSTRACT}

This study aimed to analyze the discourse of professional nurses regarding the implications for the process of work offered to the black population. This is a descriptive study with a qualitative approach, carried out between March and May 2017, based on the conceptual framework of Michel Pêcheux's discourse analysis, carried out with eight nurses from Primary Health Care in the city of Cajazeiras, Paraíba, Brazil. For data collection, an individual semistructured interview was used. The collection took place only after approval by the Research Ethics Committee. Nurses present, in their discursive corpus, that the discriminatory processes stand out in different formats, especially through prejudice and racism, and also by associating of vulnerabilities with the black population. Thus, it is essential to implement educational interventions for professionals aiming the health of the black population, breaking down historical-social barriers, and strengthening the promotion of health.

\section{RESUMEN}

Este estudio objetivó analizar el discurso del profesional enfermero acerca de las implicaciones en el proceso laboral ofrecido a la población negra. Se trata de un estudio descriptivo, con abordaje cualitativo, realizando entre los meses de marzo y mayo de 2017, sostenido en los marcos conceptuales del análisis del discurso de Michel Pêcheux, realizado con ocho enfermeros de la Atención Primaria a la Salud del municipio de Cajazeiras, Paraíba, Brasil. Para la coleta de datos fue utilizada una entrevista semiestructurada de manera individual. La coleta ocurrió solamente después de la aprobación del Comité de Ética en Investigación. Los enfermeros presentan en su corpus discursivo que los procesos discriminatorios se destacan a partir de formas diversas, en especial a través del prejuicio y racismo y, sobre todo, por la asociación de las vulnerabilidades con la población negra. De esa manera, es fundamental la implementación de intervenciones educativas sobre la salud de la población negra para los profesionales, rompiendo barreras histórico-sociales y fortaleciendo la promoción de la salud.

\section{INTRODUÇÃO}

Com a diversificação da população brasileira, o termo igualdade vem sendo utilizado de forma imprecisa quando relacionado aos cuidados ofertados à população negra, uma vez que profissionais da saúde acabam atrelando suas ações, de modo equivalente, a todas as populações, o que acaba deixando, por vezes, os cuidados específicos a essas pessoas negligenciados.

A saúde de grupos étnico-raciais em situações vulnerabilizantes evidencia as iniquidades assistenciais pelo adoecimento precoce, ou a progressão agressiva dos processos de adoecimento, assim como os elevados índices de morbimortalidade. A vulnerabilidade no processo saúde-doença da população negra brasileira resulta de um contexto histórico-social de privação de direitos humanos ${ }^{1}$.

Embora as discriminações em serviços de saúde possuam pauta em processos raciais, alguns estudos evidenciam um conjunto de fatores que influenciam além da raça nessas situações, a exemplo das discriminações por características identitárias².

Mesmo com a visivel diferença entre a ocupação de brancos e negros nos diversos espaços sociais, o racismo ainda é invisivel aos olhos da sociedade ${ }^{3}$, sendo ponto crucial na reprodução e manutenção dos processos de desigualdades raciais.

0 enfrentamento para tais situações demanda 0 desenvolvimento de teorias, práticas e técnicas em todos os cenários de assistência direta ou indireta. Porém, as formulações de ações que visam reduzir as desigualdades sociais em quesitos raciais e de gênero, em sua grande maioria, iniciam o seu desenvolvimento fora dos sistemas de saúde, por meio dos indivíduos negros em movimentos organizados, com levantamento de análises e conhecimento social 4 .

Analisando o contexto de saúde, a população negra esbarra em ineficiências de programas e ações de serviços, além do despreparo da formação de profissionais capacitados para a assistência em um cuidado integral a essa população. Acrescentandose que as pessoas negras também têm menos probabilidade de ter acesso aos cuidados de saúde ${ }^{5}$.

Isso pode ser identificado como a dimensão do racismo que migra do racismo interpessoal para a dimensão estrutural da assistência, afetando a organização, 0 atendimento, as práticas, condicionando resultados desiguais de atendimentos, caminhando para um racismo sistêmico e excludente ${ }^{4}$.

Os serviços de saúde, quando não bem preparados, reproduzem situações de cunho discriminatório, ferindo o direito do usuário perante o acesso livre de 
preconceitos e discriminações, como também ferem os princípios do Sistema Único de Saúde (SUS), que defende um sistema igualitário e equitativo para seus usuários².

Dessa maneira, o Ministério da Saúde, por meio da Política Nacional de Saúde Integral da População Negra (PNSIPN), reconhece e assume a necessidade da criação de meios para serem utilizados na promoção da saúde integral da população negra e do enfrentamento ao racismo institucional no SUS, objetivando a superação das barreiras estruturais e habituais que refletem negativamente nos indicadores de saúde dessa população, como a precocidade dos óbitos, altas taxas de mortalidade materna e infantil, maior prevalência de doenças crônicas e infecciosas e altos índices de violência ${ }^{6}$.

Neste contexto, um dos profissionais que podem desenvolver os cuidados alinhados às reais necessidades de saúde para a população negra, quebrando o paradigma da desigualdade assistencial, é o profissional enfermeiro. Este profissional, ao longo das políticas públicas que foram surgindo, ganhou cada vez mais autonomia em seu processo de trabalho, possuindo atribuições educativas, organizativas, administrativas e, principalmente, de assistência direta, promovendo o cuidado mais abrangente ao sujeito, família e comunidade no espaço da Atenção Primária à Saúde (APS) ${ }^{7}$.

Com isso, a realização de pesquisas que possam evidenciar as situações que produzem um processo de desigualdade assistencial, assim como a atuação no cuidado de enfermeiros da APS, fazem-se necessárias pela realização da identificação das lacunas assistenciais e por possibilitarem apontar possiveis caminhos para prevenir ou solucionar tais problemáticas.

Dessa forma, surgiu a seguinte questão norteadora: Qual é o discurso do profissional enfermeiro acerca das implicações no processo de trabalho ofertado à população negra?

Logo, esta investigação objetiva analisar o discurso do profissional enfermeiro acerca das implicações no processo de trabalho ofertado à população negra.

\section{METODOLOGIA}

Este estudo tem natureza descritiva e abordagem qualitativa. Foi realizado na APS do município de Cajazeiras, no estado da Paraíba, Brasil. Essa cidade faz parte da $4^{a}$ Macrorregião de Saúde e da $9^{a}$
Gerência Regional de Saúde da Paraíba.

Fazem parte do cenário de estudo 17 enfermeiros que compõem as 23 Equipes de Saúde da Família do município de Cajazeiras, que atualmente integram 19 Unidades Básicas de Saúde (UBS).

0 critério de inclusão utilizado foi trabalhar há mais de 12 meses como enfermeiro na APS, compreendendo-se que esse seja período satisfatório para estabelecer o vínculo com a dinâmica desse cenário de atenção. Foram adotados como critérios de exclusão: estar de férias, de licença-saúde ou afastado do serviço.

Após utilizar os critérios de inclusão e exclusão, foram convidados 13 profissionais; destes, cinco se recusaram a participar com as justificativas de que não gostariam de falar sobre o assunto, não tinham domínio sobre o tema ou não gostavam de realizar entrevistas gravadas. Totalizando oito enfermeiros como participantes do estudo.

Para a análise de discurso, não se considera o quantitativo de participantes, e sim a análise em profundidade dos discursos proferidos. 0 encerramento das entrevistas ocorreu a partir do momento em que aconteceu a saturação teórica, pois não houve acréscimo de novas informações.

A coleta de dados ocorreu entre os meses de março e maio de 2017 e foi realizada por meio de uma entrevista individual semiestruturada que continha perguntas discursivas voltadas à compreensão dos enfermeiros acerca da saúde da população negra, das práticas cuidativas específicas a essa população e de sua realização na rotina assistencial.

Primeiramente, as entrevistas eram gravadas por meio do aparelho celular, após a autorização por escrito do participante, e tiveram duração média de oito minutos. Após as entrevistas, estas foram escutadas diversas vezes para a melhor análise e compreensão das falas para a sua transcrição.

A metodologia analítica utilizada nesta investigação foi a Análise de Discurso (AD), a qual favorece o reconhecimento da significância da linguagem. É a partir da linguagem que o homem forma e transforma sua história. Nesse sentido, a $A D$ avalia não somente o que foi dito, mas também as condições nas quais aquilo foi dito, ou seja, a linguagem é influenciada por sua exterioridade sem jamais ignorar a sua historicidade ${ }^{8}$.

Assim, neste estudo, seguiram-se, com base na $A D$, três etapas que favorecem o reconhecimento da significância de um discurso, sendo elas: passagem da superfície linguística para o objeto discursivo; 
passagem do objeto discursivo para o processo discursivo e passagem do processo discursivo para a formação ideológica8.

A investigação foi iniciada após a aprovação do projeto pelo Comitê de Ética em Pesquisa da Universidade Federal de Campina Grande, campus de Cajazeiras, sob o parecer n. ${ }^{\circ} 2.012 .785 / 2017$ e CAAE 65779517.1.0000.5575. A participação na pesquisa ocorreu mediante a assinatura, por parte do entrevistado, do Termo de Consentimento Livre e Esclarecido. Foram respeitados todos os preceitos éticos e legais trazidos na Resolução 466/2012 do Conselho Nacional de Saúde ${ }^{9}$, em que se garantiu o anonimato dos participantes, utilizando a sigla ENF, seguida da numeração conforme ordem das entrevistas.

\section{RESULTADOS E DISCUSSÃO}

Após a análise dos discursos, obtiveram-se duas categorias: Racismo e preconceito e Associação das vulnerabilidades sociais com a população negra.

\section{Racismo e preconceito}

Em primeiro momento, é necessária uma abordagem sobre a postura dos entrevistados quando questionados sobre a saúde da população negra, pois, a princípio, não existiam questões que propusessem o diálogo sobre esses temas; porém, nas respostas obtidas, a maioria enfatizava sobre questões de preconceito e racismo contra a população negra.

Eu acho que sim, não é uma coisa muito divulgada, né? Acho que esbarra muito também no medo do crime de racismos, então, assim, é muito delicado, porque nem você não pode ser ou expressar atos racistas, né? (ENF 04).

Preconceito existe? Existe! Pode ter dentro de alguns enfermeiros? Pode! Entendeu? Mas que mesmo que tenha, não transpareça, entendeu? (ENF 06).

Isso existe muito, né? Aqui na unidade, graças a Deus não tivemos esse problema, mas existe vários. Vários relatos, a gente vê artigos, revistas, entrevistas que a pessoa foi chamada... Que o profissional chamou de negro, negrinho e tudo mais, isso é bem comum, infelizmente nos dias de hoje, né? (ENF 03).

Na primeira análise, percebe-se o tabu que se encontrava no ambiente da entrevista, o desconforto em dialogar sobre algo que não deve ser dito, pois não existe; que não pode ser expresso, pois é "crime"; que não pode ser considerado presente, pois "a lei caindo mais em cima" propõe o misticismo de se falar sobre população negra. Embora discursos preconceituosos e racistas sejam muitas vezes ecoados, a naturalização das práticas ainda se mantém de forma camuflada.

Para muitos, o significado de preconceito e racismo vincula-se à violência verbal ou física relacionada à cor negra, dissipando outras formas de atuação que também poderiam ser consideradas como algo racista ou preconceituoso, a naturalização de que a violência por raça se restringe a agressões físicas e verbais, desconsiderando as iniquidades assistenciais.

Pode-se abordar ainda nesse contexto do dito/ não dito os silenciamentos existentes de forma sutil nos discursos. Deve-se considerar em um silenciamento o subentendido dos dizeres, é algo que não foi expresso em um discurso, mas encontrase nas entrelinhas dos dizeres, o que depende do contexto, e, necessariamente, esses subentendidos também significam em uma análise ${ }^{10}$.

Para muitos, não existem formas plausiveis de enfrentar o preconceito racial, e muito menos buscam-se formas de evitá-lo, pois é mais simples manter a vergonha de se admitir o preconceito do que atitudes que visem interferir na realização de ações discriminativas ${ }^{11}$.

Ainda de acordo com Lins, Nunes e Camino ${ }^{11}$, as hierarquias sociais permanecem de forma cristalizada, perpetuando uma amabilidade às questões sociais relacionadas aos negros, e evitando demonstrar ou falar algo que venha a quebrar a identidade social de realização de atos discriminatórios.

No que se refere à atuação dos profissionais da saúde, especificamente os enfermeiros atuantes na APS, existe a falta de questionamentos que instiguem a problematização social, em que somente por meio de um cuidado à saúde que busque este profissional para reflexões críticas é que torna-se possivel uma assistência de equidade, tratando os desiguais de forma desigual, pois uma visão ampla significa uma atuação ampla.

Demonstrando a importância desse tema, uma 
pesquisa-ação sobre as desigualdades raciais, realizada em São Paulo, conseguiu incluir com os profissionais de saúde a necessidade de ver as desigualdades presentes dentro das desigualdades, e explicar o que as implicações de um atendimento inadequado, ineficaz e discriminatório pode causar no sistema de saúde ${ }^{12}$.

Tratando-se de políticas públicas no Brasil, o principal objetivo termina por tentar minimizar as disparidades em saúde, evidenciando o contexto social e político com as influências nos aspectos de vida de um grupo populacional, no caso da Política Nacional de Saúde Integral da População Negra ${ }^{6}$.

Porém, para a boa execução dessa política, fazse necessário reconhecer e quebrar paradigmas, como a discriminação e o racismo. 0 racismo acaba promovendo o acesso restrito à assistência, diminuindo, consequentemente, a participação ativa da população negra em práticas saudáveis e limitando a entrada nos serviços de saúde, ocasionando mais mortes por causas evitáveis ${ }^{13}$.

\section{Associação das vulnerabilidades sociais com a população negra}

Neste discurso, é possivel observar outra esfera da realidade social enfrentada por negros e negras no Brasil, principalmente se residirem em áreas mais carentes e periferias.

Agora, se fosse em outras comunidades, talvez teria, entendeu? Não pelo, pela cor, por ser pela cor, mas pelo, pela que às vezes eles são meio, são meio, são desinformados! Entendeu? Porque lá, é uma população muito carente, entendeu? E lá tem bastante, negros, entendeu? (ENF 06).

Percebe-se a associação das vulnerabilidades sociais com a população negra, de modo que, pelo discurso dos enfermeiros, seria possível ter uma atuação diferenciada em determinados locais onde ocorre maior fragilidade.

Os processos de desigualdades relacionam-se a fatores sociais hierarquizados, resultando não apenas em fatores individuais, mas, principalmente, na forma como uma sociedade encontra-se organizada, por valores simbólicos e materiais, possibilitando a partilha desigual movida por processos demográficos, sociais, econômicos, culturais e políticos, proporcionando, com isso, as vantagens e desvantagens sociais em saúde presentes no mundo ${ }^{14}$.

Comparando-se com a população norte-americana, em termos territoriais, há bairros onde a população é predominantemente negra. Nesses territórios, também se encontra uma população vulnerável, exposta a poluentes e toxinas, além do difícil acesso ao sistema educacional e à saúde ${ }^{15}$.

Pode ser classificado o discurso como uma estereotipação da pessoa negra residente na área citada, assim como também é possivel discutir sobre o impacto causado pela situação econômica e social apresentada pela população negra no Brasil. 0 profissional, ainda que de forma relutante, relaciona essa diferenciação social quando expõe a localidade e a situação frequentemente encontrada naquela comunidade citada.

Embora não seja admitido, na maioria dos casos a formação da imagem coloca a pessoa negra em situações de vulnerabilidade social, econômica e educacional, que nada mais é do que o retrato do Brasil, porém camuflado pela venda da ideia de democracia racial ${ }^{3}$.

Quando são tratados temas que envolvem a população negra, de forma inconsciente e automática existe uma formação da imagem sobre o que é ser negro no Brasil, e, mesmo que dito de forma relutante, é quase palpável o desconforto em evidenciar tal fato pelo profissional quando fala que a população mais vulnerável é negra e desinformada.

Essa fala pode estar interligada aos resultados de uma análise de desigualdades sociais por cor e raça do Instituto Brasileiro de Geografia e Estatística (IBGE), demonstrando que, no ano de 2018, o percentual obtido para o analfabetismo entre negros e brancos evidenciou que os brancos com 15 anos ou mais apresentavam $3,9 \%$ de analfabetismo; em contrapartida, os negros, considerando a classificação parda e preta, apresentavam um valor de 9,1\% de analfabetismo para essa mesma faixa etária ${ }^{16}$. Reafirmando, portanto, uma posição desfavorável e vulnerabilizante para a parcela populacional considerada negra.

A palavra "desinformado" percorre o discurso carregada de significados quando analisada, significados esses que podem interferir de forma negativa na atuação do profissional enfermeiro à pessoa negra que venha a utilizar os serviços ofertados pela APS.

É evidente no texto que, quem reside em determinada região é carente e desinformado, e dependendo do contexto crítico e reflexivo do 
profissional, a sua atuação pode interferir de forma a individualizar negativamente 0 atendimento, entendendo que a desinformação não seja digna de um empoderamento, o que resulta em prognósticos negativos à saúde.

0 obstáculo em perceber situações de discriminação no acesso à saúde e de racismo no dia a dia do serviço perpassa as lacunas não preenchidas no processo formativo, uma vez que os profissionais também fazem parte do sistema social e, dessa forma, estão sujeitos às questões representacionais históricas em relação ao negro, o que pode acarretar no pensamento de democracia racial, que encobre os determinantes e condicionantes da saúde da população negra e sua relação com o ambiente social, afetando diretamente as condições e qualidade de vida dessas pessoas ${ }^{17}$.

Dessa maneira, é necessário garantir um atendimento ético, humanizado e de qualidade, por meio de iniciativas que garantam capacitação para os profissionais de saúde para que possam executar a PNSIPN com cursos voltados para gestores, profissionais e sociedade civil, abrangendo temas que englobem os condicionantes e determinantes de saúde. Além de reforçar o trabalho na promoção da equidade e enfrentamento à discriminação nas instituições e serviços do SUS ${ }^{18}$.

\section{CONCLUSÃO}

Os achados deste estudo evidenciaram que, na visão dos enfermeiros, os processos discriminatórios destacam-se de diversas formas, por vezes de forma consciente, por vezes de forma inconsciente, principalmente por meio do preconceito e racismo e, ainda, pela associação das vulnerabilidades à população negra.

A construção da saúde no Brasil possui peculiaridades relacionadas ao contexto sociocultural com a sua história, em que uma sociedade rodeada de pudores e misticismos para os debates raciais resulta em forte barreira para a efetivação de assistência equitativa, produzindo uma fragmentação assistencial.

Uma das principais limitações encontradas no desenvolvimento do presente estudo foi conseguir dialogar com profissionais de enfermagem sobre a saúde da população negra, os próprios limites pessoais de cada um transformaram-se em lacunas, expondo o medo de falar sobre tal tema e, principalmente, o medo de pôr em prática socialmente as diretrizes da política à saúde negra, o que pode produzir resultados sociais insatisfatórios, aumentado os riscos e diminuindo a assistência.

Sugere-se que se desenvolvam novas pesquisas que contribuam para reflexões na assistência à saúde integral dessa população, com ênfase na intervenção educativa dos enfermeiros e da equipe de saúde multiprofissional, uma vez que é necessário reconhecer a importância das ações de saúde, diminuindo barreiras históricas, promovendo a equidade e fortalecendo a promoção da saúde a essa população.

\section{CONTRIBUIÇÃO DOS AUTORES}

Maísa Pereira Galdino realizou a concepção do projeto, coleta, análise, interpretação dos dados e redação do artigo. Daniele Pereira Soares, Cícera Renata Diniz da Silva, Dayze Djanira Furtado de Galiza e Mayara Evangelista de Andrade realizaram a interpretação dos dados, redação do artigo e revisão final. Marcelo Costa Fernandes realizou a concepção e delineamento, análise e interpretação dos dados, redação do artigo e aprovação da versão a ser publicada.

\section{REFERÊNCIAS}

1. Prestes CRS, Paiva VSF. Abordagem psicossocial e saúde de mulheres negras: vulnerabilidades, direitos e resiliência. Saúde Soc. [serial on the internet ]. 2016 Set [cited 2021 Jan 21]; 25(3); 673-88. Available from: https://www.scielo.br/pdf/sausoc/ v25n3/1984-0470-sausoc-25-03-00673.pdf

2. Bastos JL, Garcia LP. Discriminação nos serviços de saúde. Epidemiol Serv Saúde [serial on the internet ]. 2015 [cited 2021 Jan 21]; 24(3):351-52. Available from: https://www.scielosp.org/article/ ress/2015.v24n3/351-352/pt/

3. Oliveira BMC, Kubiak F. Racismo institucional e a saúde da mulher negra: uma análise da produção científica brasileira. Saúde debate [serial on the internet ]. 2019 [cited 2021 Jan 21]; 43(122):93948. Available from: https://www.scielosp.org/ article/sdeb/2019.v43n122/939-948/pt/

4. Werneck J. Racismo institucional e saúde da população negra. Saúde Soc [serial on the internet ]. 2016 [cited 2021 Feb 3]; 25(3):535-49. Available from: https://www.scielo.br/pdf/sausoc/ v25n3/1984-0470-sausoc-25-03-00535.pdf

5. Lancet. Racial inequities in HIV. Editorial. 2020; 7(E449). doi: https://doi.org/10.1016/S2352$\underline{3018(20) 30173-9}$ 
6. Brasil. Ministério da Saúde. Política Nacional de Saúde Integral da População Negra: uma política para o SUS. Brasília (DF): Editora do Ministério da Saúde; 2017.

7. Barros RC, Silva AFL, Maia ISL, Silva LBS. Atuação do enfermeiro na atenção primária à saúde no município do Rio de Janeiro. Saúde em Redes [serial on the internet]. 2020. [cited 2021 Nov 16]; 6(3):157-71. Available from: http://revista. redeunida.org.br/ojs/index.php/rede-unida/article/ view $/ 2918 / 589$

8. Silva RS, Silva GV, Bressanin JA. Entre paráfrase e polissemia: a movência dos sentidos e dos sujeitos em "saímos do Facebook". Entrepalavras [serial on the internet]. 2017 Nov [cited 2021 Jan 5]; 7(2):22942. Available from: http://www.entrepalavras. ufc.br/revista/index.php/Revista/article/ view $/ 764 / 456$

9. Brasil. Resolução n. ${ }^{\circ}$ 466, de 12 de dezembro de 2012. Diário Oficial da União [document on the internet]. 2012 [cited 2021 Nov 16]; Seção 1. Available from: https://conselho.saude.gov.br/ resolucoes/2012/Res0466.pdf

10. Orlandi EP. Análise de discurso: princípios e procedimentos. 11. ed. Campinas: Pontes; 2013.

11. Lins SLB, Nunes AL, Camino L. 0 papel dos valores sociais e variáveis psicossociais no preconceito racial brasileiro. Psicol Soc [serial on the internet ]. 2014 [cited 2021 Jan 5]; 26(1):95-105. Available from: https://www.scielo.br/pdf/psoc/v26n1/11.pdf

12. Batista LE, Rattner D, Kalckmann S, Oliveira MCG. Humanização na atenção à saúde e as desigualdades raciais: uma proposta de intervenção. Saúde Soc [serial on the internet]. 2016. [cited 2021 Jan 5]; 25(3):689-702. Available from: https://www.scielo.br/scielo.php?script=sci arttext\&pid=S0104-12902016000300689\&lng=en.

13. Mendes AGS, Eduardo GN. Vidas Negras Importam! Um estudo sobre os indicadores sociais da mortalidade materna de mulheres negras na Paraíba. Cad gêneros e diversidade [serial on the internet]. 2020 [cited 2021 Jan 10]; 6(1):69-83. Available from: https://periodicos.ufba.br/index. php/cadgendiv/article/view/27817/20816

14. Beech BM, Ford C, Thorpe RJ Jr, Bruce MA, Norris KC. Poverty, Racism, and the Public Health Crisis in America. Front Public Health [serial on the internet]. 2021 [cited 2021 Jan 21]; 9:699049. Available from: https://doi.org/10.3389/fpubh.2021.699049

15. Bailey ZD, Krieger N, Agénor M, Graves J, Linos N, Bassett MT. Structural racism and health inequities in the USA: evidence and interventions. Lancet. 2017; 389(10077):1453-63. Available from: https://
doi.org/10.1016/S0140-6736(17)30569-X

16. Instituto Brasileiro de Geografia e Estatística. IBGE. Desigualdades Sociais por Cor ou Raça no Brasil [homepage on the internet]. Estudos e Pesquisas. Informação Demográfica e Socioeconômica. 2019 [cited 2021 Jan 15]; n. 41. Disponível em: https://biblioteca. ibge.gov.br/visualizacao/livros/liv101681 informativo.pdf

17. Rosa LGF, Christóvão RG, Furlin M, Lasta JB. Nurses perceptions and actions about institutional racism in public health. Rev Enferm UFSM [serial on the internet]. 2019 [cited 2021 Feb 15]; 9(8):1-19. Available from: https:// periodicos.ufsm.br/reufsm/article/view/31131/ pdf 1

18. Tavares HHF, Moraes BA, Matias AG, Silva HBS, Bernardo LNG. Análise e perspectiva sobre a formação do profissional de saúde para o atendimento à mulher negra. Extension: R Eletr Extensão [serial on the internet]. 2018 [cited 2021 March 3]; 15(28):19-28. Available from: https://periodicos. ufsc.br/index.php/extensio/article/view/1807$\underline{0221.2018 v 15 n 28 p 19 / 36391}$
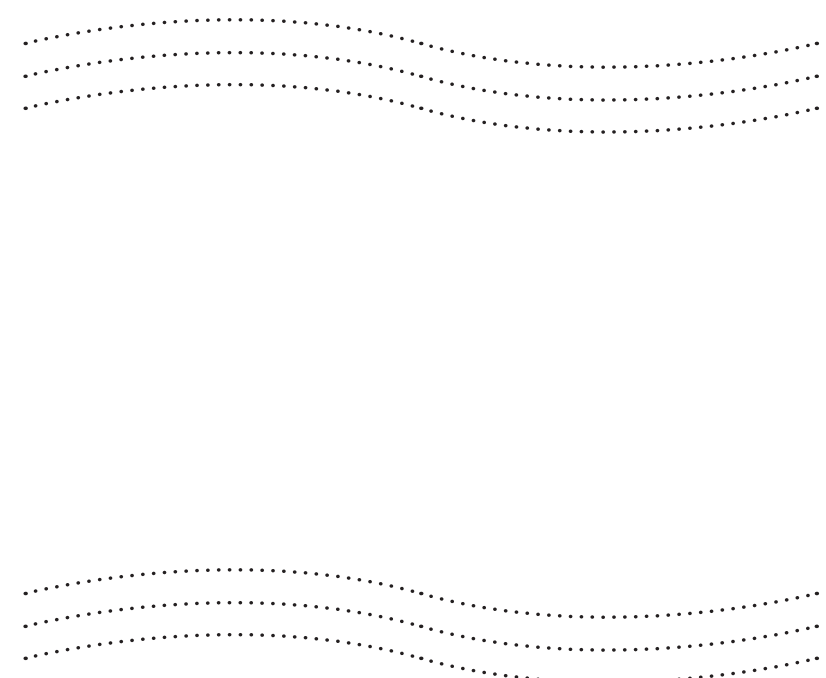